\title{
Incorporação de Matemática Fuzzy em Métodos Multicriteriais para Descrever Critérios Subjetivos em Planejamento de Recursos Hídricos: Fuzzy - CP e Fuzzy - CGT
}

\author{
Antonio Carlos Zuffo \\ Faculdade de Engenharia Civil, Arquitetura e Urbanismo - FEC/UNICAMP \\ zuffo@fec.unicamp.br
}

Recebido: 25/07/09 - revisado: 30/03/10 - aceito: 15/08/11

\section{RESUMO}

Em muitos estudos ambientais e de planejamento e gestão de recursos hídricos há a necessidade de se considerar critérios com poucos dados ou cuja interpretação depende do conhecimento de especialistas. Por esse motivo, critérios incorporam a subjetividade, cuja representação por números escalares reduz sua tradução fidedigna. Critérios ambientais, sociais, culturais ou daqueles que dependem de uma estimativa ou previsão carregam graus de imprecisão, ou incerteza quanto ao seu real valor, mas que podem perfeitamente estar contidos em uma faixa de possibilidades, com diferentes faixas de valores ou probabilidades. Essa graduação pode ser traduzida pelas funções de pertinência dos números fuzzy, o que poderá possibilitar uma melhor performance das análises multicriteriais nessas aplicações. Este trabalho propõe a incorporação da aritmética fuzzy em dois métodos multicriteriais muito utilizados em planejamento e gestão de recursos hídricos, os métodos "Compromise Programming" (CP) e "Cooperative Game Theory" (CGT), que deram origem ao Fuzzy-CP e ao Fuzzy-CGT. A aritmética fuzzy adotada é aquela proposta para números fuzzy representados por funções de pertinência triangulares. Esses métodos adaptados possibilitaram a adoção de critérios abstratos ou com melhor representação de seus possíveis intervalos de variação, contribuindo para uma melhor interpretação do problema, como também do reconhecimento de sua fragilidade, mostrando-se viáveis para resolução de problemas dessa natureza.

Palavras-chave: Planejamento de recursos hídricos, métodos multicriteriais fuzzy, análise multicriterial.

\section{INTRODUÇÃO}

As ferramentas de planejamento são desenvolvidas na medida em que vão surgindo novas demandas ou se as limitações das ferramentas disponíveis não mais podem ser aceitas, devido à necessidade de incrementar o processo decisório.

No Brasil, até o final do século XX, por meio do Código da Águas de 1934, foi adotado o modelo burocrático de gerenciamento de recursos hídricos. Nessa época, excetuando-se o sertão nordestino, os recursos hídricos no Brasil eram abundantes, em quantidade e em qualidade. As recentes descobertas dos processos de tratamentos químicos, para o período, davam a segurança de que qualquer água, não importando o quanto fosse poluída, poderia ser tratada. Essa falsa crença causou uma falta de compromisso com a preservação das áreas de mananciais, que de certa forma eram protegidas até então, heranças das "Ordenações Manoelinas e Alfonsinas" do período colonial.
A análise benefício/custo foi uma das ferramentas mais utilizadas em todos os tipos de análises de viabilidade econômica. Nos Estados Unidos, na década de 1930 no auge da grande depressão econômica, surgiu outro modelo de gerenciamento de recursos hídricos. $\mathrm{O}$ modelo econômicofinanceiro, aplicado no Vale do Rio Tennessee (TVA - Tennessee Valley Authority), que visava promover o desenvolvimento regional, fazendo uso de instrumentos econômicos e financeiros aplicados pelo poder público, na região mais carente e atrasada dos EUA. Objetivava acelerar a economia de uma região com os maiores índices de desemprego e de falta de qualificação profissional de todos os EUA. Esse modelo foi adotado pelo governo brasileiro para a bacia do rio São Francisco, por meio da CODEVASF (Companhia de desenvolvimento dos Vales do São Francisco e do Parnaíba). Nesse modelo a análise benefício/custo impera e tem a característica de, em pouco tempo, induzir o desenvolvimento econômico. Deve ser utilizado em períodos curtos, pois pode levar à degradação ambiental se não existir legislação ambiental que imponha limites e restrições, 
uma vez que seu objetivo prioritário é o crescimento econômico, em detrimento dos demais.

Nesse modelo de gerenciamento já era reconhecida a limitação ou insuficiência dessa análise benefício/custo para decidir sobre investimento em infra-estrutura hidráulica que envolvesse milhões de reais e que podem causar grandes impactos, tanto positivos quanto negativos.

Nas décadas que se sucederam à promulgação do "Código das Águas" observou-se que a capacidade de criação de novos compostos químicos era muito maior que a nossa capacidade de acompanhálos com novas técnicas de tratamento economicamente viáveis. Observou-se passivamente o aumento da poluição dos rios, como o discurso oficial de que esse era o preço a ser pago pelo desenvolvimento econômico do País.

A avaliação de projetos de recursos hídricos, considerando apenas o critério econômicofinanceiro levou a diferentes graus de degradação do meio-ambiente, devido a carência de legislação ambiental, que teria a função de restringir e limitar ações que provocassem a degradação ambiental.

O Brasil vive hoje outra realidade, a partir da publicação da Lei 9.433, de 8 de janeiro de 1997, conhecida como a Lei das Águas. O modelo de gerenciamento dos recursos hídricos passou a ser o Modelo Sistêmico de Integração Participativa (MSIP).

Com a adoção do MSIP de gerenciamento, mudou-se o paradigma, e não mais se pode considerar apenas um uso prioritário, avaliado por um único critério na análise de viabilização de projetos de infra-estrutura de recursos hídricos. A geração de energia elétrica deixou de ser o uso prioritário, como foi por mais de 60 anos.

Um dos fundamentos básicos desta Lei trata da necessidade de ser proporcionado o uso múltiplo das águas, priorizando-se o consumo humano e a dessedentação de animais em caso de escassez. A água passou a ser uma commodity muito disputada, e necessária para todo o tipo de atividade antropogênica. Por essas razões, é unânime o reconhecimento de que critérios ambientais, sociais e culturais devam ser considerados conjuntamente com os critérios econômicos para a tomada de decisão nesse novo paradigma.

A análise multicriterial tem mostrado que é uma ferramenta poderosa na avaliação de projetos e seleção de cenários em diferentes fases do planejamento, principalmente daqueles que envolvam múltiplos decisores, ambiente e sociedade, requeridos pelo MSIP.
No Brasil ainda é precário o reconhecimento das possibilidades de associação, entre o planejamento multicriterial e as valorações ambiental e social, sejam técnica e ambientalmente sustentáveis, para assegurar que a tomada de decisão sobre recursos hídricos torne-se mais eficaz. Nos últimos anos, diversas pesquisas foram realizadas no sentido de aplicar a análise multicritério ao planejamento de recursos hídricos. Com isso, alguns estudos já vêm incorporando critérios sociais e ambientais às análises multicritério.

Muitos técnicos, no entanto, apesar de reconhecerem a necessidade da incorporação de critérios sociais e ambientais, admitem que essa incorporação não é tarefa fácil conforme apontam Jardim e Lanna (2003), Boclin e Mello (2006), Munier (2006).

As estimativas tradicionais de análises de Benefício/Custo, que procuram incorporar alguns custos e benefícios indiretos, determinados por diferentes métodos, tais como: DCEs (Discrete Choice Experiments), MAC (Método de Avaliação Contingente), MPH (Método dos Preços Hedônicos), MCV (Método dos Custos de Viagem), e CR (Custo de Reconstrução), entre outros. Os tão conhecidos custos e/ou benefícios intangíveis, mostraram-se deficientes, e muitas vezes não passaram de estimativas grosseiras. Estimativas essas geradoras das maiores críticas por parte do meio técnico, pois cada metodologia estima valores monetários diferentes para o mesmo indicador (Sharratt (1999), Munda (2008), Ergas e Robson (2009), Hoyos (2010), entre tantos outros), chegando a ser chamada, inclusive de técnica de adivinhação por Eustácio e Távora Júnior (1999).

Os critérios sociais e ambientais geralmente são de difícil representação matemática, pois, muitas vezes expressam apenas a subjetividade de seus avaliadores, e que variam de profissional para profissional, uma vez que as opiniões podem ser diversas, e as diferentes metodologias induzem a diferentes resultados.

A subjetividade não é vista com bons olhos no meio técnico para ser utilizada em análises de viabilidade devido a sua natureza abstrata. É difícil ou mesmo impossível a representação matemática de algum objetivo considerado abstrato e, por consequência, as técnicas de otimização não podem ser utilizadas para resolver esses problemas.

A matemática fuzzy, por sua vez, é capaz de representar a subjetividade, que alguns critérios sócio-ambientais possam apresentar, com maior propriedade que a matemática tradicional (Zuffo, 2009). Um critério fuzzy define uma faixa de valores 
possíveis para um determinado critério difuso - não representa um número exato, mas um intervalo de números possíveis - definido por um valor mais provável e pelos seus limites de variação (função de pertinência).

A representação de alguns critérios subjetivos por meio de funções fuzzy, não garante por si só sua aplicação, há ainda a necessidade do desenvolvimento de métodos multicriteriais capazes de trabalhar com essa nova definição de critérios e aritmética fuzzy.

O desenvolvimento dessas ferramentas ainda é necessário, uma vez que a própria matemática fuzzy não se encontra totalmente desenvolvida. Assim sendo, este artigo apresenta uma proposta de modificação de dois métodos multicriteriais bastante simples, baseados na distância da solução avaliada em relação à solução ideal, para permitir o cômputo com critérios representados por números fuzzy.

A aritmética fuzzy proposta por Dubois e Prade (1978, 1979 e 1984) foi incorporada em dois métodos multicriteriais, o CP (Compromise Programming) e o CGT (Cooperative Game Theory), dando origem a suas versões fuzzy.

\section{NÚMEROS FUZZY TRIANGULARES}

Nos métodos CP e CGT o desempenho de cada cenário para cada critério considerado é representado por um número fuzzy. Isso porque, na maioria dos casos, a entrada de dados pode não ser definida dentro de um grau razoável de precisão. Muitos dos critérios são avaliados de maneira a incorporar a subjetividade ou probabilidades, em que uma representação estocástica pode representá-la (Zuffo, 2001).

Os números fuzzy são apresentados de acordo com a proposta de Dubois e Prade (1978), da mesma maneira que foram utilizados por Goumas e Lygerou (2000) e Zuffo (2009) na seguinte forma: $x=(m, a, b)_{E D}$, em que os valores menores que $(m-a)$ e maiores que $(m+b)$ não pertencem ao número fuzzy, e possuem a função de pertinência igual a zero $f(x)=0$. Para valores compreendidos entre $(m-a)$ e $(m+b)$ em que a função de pertinência varia de grau e $0<f(x) \leq 1$, e para valor igual a $m$ a função de pertinência será máxima e $f(x)=1$. E e D representam os intervalos limítrofes, de " $m$ ", esquerdo e direito, respectivamente.

Essa representação de números fuzzy é a de forma triangular e, portanto, assumi-se uma variação linear em sua função de pertinência. No intervalo compreendido entre $m-a<x \leq m+b$ existe uma variação gradual da função de pertinência de " $x$ ", associada a um valor real compreendido entre 0 e 1 . Por exemplo, assumindo-se funções lineares para E e $\mathrm{D}$, representado pelo número fuzzy $x=(0.6,0.4,0.2)$, como representado graficamente na Figura 1 por um triângulo.

A função de pertinência de um determinado valor, dentro do intervalo desse número fuzzy pode ser determinada da seguinte maneira: o número 0.7 possui uma de pertinência de 0.5 , assim como para o número 0.4 , em que a pertinência também corresponde a 0.5 .

As operações básicas fuzzy utilizadas, neste artigo, são aquelas propostas por Dubois e Prade (1978, 1979 e 1988), e estão sumarizadas na Tabela 1, segundo Dubois e Prade (1978, 1979 e 1984).

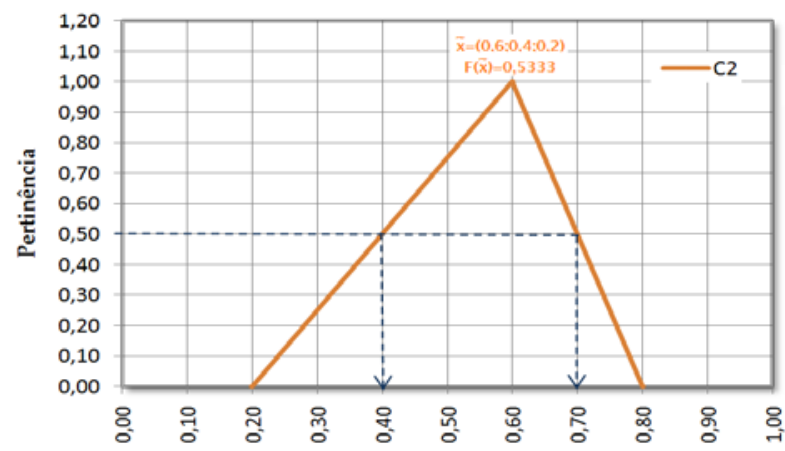

Figura 1 - Representação gráfica do número fuzzy $(0.6,0.4,0.2)_{\mathrm{ED}}$ para funções lineares $\mathrm{E}$ e D.

\section{Método - CP (Compromise Programming)}

O método CP é um dos métodos multicriteriais mais utilizados em razão de sua simplicidade matemática. Este método está baseado no conceito de distância métrica (Teorema de Pitágoras), entre dois pontos cujas coordenadas são conhecidas (Zeleny, 1982). O método procura minimizar a distância de todos os pontos factíveis avaliáveis, para um determinado ponto escolhido pelo DM (Decision Maker - Decisor ou Tomador de Decisão), chamado "ponto ideal". Uma "solução ideal" é definida pela função objetivo $f_{i}^{*}(x)=\max f_{i}(x)$. O vetor $f^{*}$ é aquele formado por todos os elementos máximos: $f^{*}=\left(f_{1}^{*}, f_{2}^{*}, \cdots, f_{n}^{*}\right)$. 
Incorporação de Matemática Fuz乏y em Métodos Multicriteriais para Descrever Critérios Subjetivos em Planejamento de Recursos Hídricos: Fuzzy - CP e Fuzzy - CGT

Propõe-se a incorporação da matemática fuzzy no método CP, fazendo-se com que os parâmetros representados por números escalares sejam substituídos por números fuzzy triangulares e a equação original é reescrita, conforme apresentado pela Equação (1).

$$
\begin{aligned}
& l_{s}\left(x, i_{S E}, i_{S D}\right)_{E D}= \\
& =\left(\sum_{i=1}^{n} \alpha_{i}\left|\frac{f_{i}^{*}\left(z, i_{z E}, i_{z D}\right)_{E D}-f_{i}\left(x, i_{x E}, i_{x D}\right)_{E D}}{f_{i}^{*}\left(z, i_{z E}, i_{z D}\right)_{E D}-f_{i w}\left(w, i_{w E}, i_{w D}\right)_{E D}}\right|\right)
\end{aligned}
$$

Em que:

$\alpha_{i}=$ pesos atribuídos subjetivamente pelo DM ou derivado de alguma estrutura de preferência;

$$
f_{i, w}\left(w, i_{w E}, i_{w D}\right)_{E D}=\text { valor mínimo obtido pelo crité- }
$$
rio

$i$ entre todas as alternativas, ou mínimo da escala; $f_{i}\left(x, i_{x E}, i_{x D}\right)_{E D}=$ valor do resultado da implementação da decisão $\mathrm{x}$ com respeito ao $\mathrm{i}_{\text {ésimo }}$ critério;

$f_{i}^{*}\left(z, i_{z E}, i_{z D}\right)_{E D}=$ valor máximo obtido pelo critério $i$ entre todas as alternativas, ou máximo da escala.

Nesta proposta, os cálculos dos intervalos, esquerdo e direito, acompanham os cálculos dos números principais, respeitando-se a aritmética prevista na Tabela 1.

\section{Método CGT (Cooperative Game Theory)}

O método da Teoria dos Jogos Cooperativos também é um método baseado na distância a uma solução indesejada. Sua aplicação é bastante simples e por isso também muito utilizado em análises multicriteriais.

O CGT ao invés de minimizar a distância de ponto ideal, a "melhor solução" é aquela que maximiza a distância de algum ponto "status quo" de nível mínimo, em que a medida de distância utilizada é a geométrica (Gershon e Duckstein, 1983).

Apresenta-se uma proposta de incorporação da metodologia fuzzy no método CGT similar àquela proposta pelo método Fuzzy-CP. Com a introdução de números fuzzy triangulares na estrutura do método CGT, a equação original pode ser reescrita na forma da expressão (2): $l_{s}\left(x, i_{S E}, i_{S D}\right)_{E D}=\prod_{i=1}^{n}\left|f_{i}\left(x, i_{x E}, i_{x D}\right)_{E D}-f_{i}^{*}\left(z, i_{z E}, i_{z D}\right)_{E D}\right|^{\alpha_{i}}$

Em que:

$\alpha_{i}=$ é o peso do $\mathrm{i}_{\text {ésimo }}$ critério;

$f_{i}^{*}\left(z, i_{z E}, i_{z D}\right)_{L D}=$ é o $i_{\text {ésimo }}$ elemento do ponto "status quo";

$f_{i}\left(x, i_{x E}, i_{x D}\right)_{E D}=$ é o resultado da implementação da decisão x com respeito ao $i_{\text {ésimo }}$ critério;

Para ambos os métodos os critérios ambientais e sociais foram descritos por números fuzzy. A performance final de cada alternativa, para cada um dos métodos, retornou uma distância também descrita por um número fuzzy.

Somente após sua transformação para um número escalar é que a comparação dos resultados e a determinação final se tornam possíveis.

Todos os critérios foram representados por números fuzzy, mesmo para os critérios que possuíssem intervalos limítrofes, esquerdos e direitos, iguais a zero.

A transformação fuzzy-escalar utilizada é aquela proposta por Yager (1981), que corresponde a projeção do centro do triângulo formado pelo número fuzzy, conforme apresentado a seguir e ilustrado pelas Figuras 2 e 3:

$F(m, a, b)=\frac{(3 m-a+b)}{3}$
$F(0,6 ; 0,4 ; 0,2)=\frac{(3 * 0,6-0,4+0,2)}{3}=0,5333$

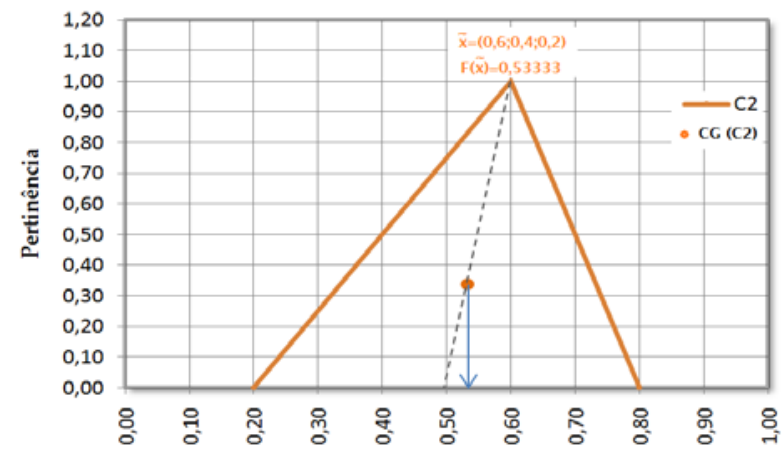

Figura 2 - Valor escalar do \# fuzzy $(0.6 ; 0.4 ; 0.2)$, (Yager, 1981). 
Tabela 1 - Operações aritméticas para números fuzzy $(\mathbf{m}, \mathbf{a}, \mathbf{b})_{\mathrm{ED}}$ : Fonte: Modificado de Goumas e Lygerou (2000).

\begin{tabular}{|c|c|}
\hline Adição & $(m, a, b)_{E D} \oplus(n, c, d)_{E D}=(m+n, a+c, b+d)_{E D}$ \\
\hline Oposto negativo & $-(m, a, b)_{E D}=(-m, b, a)_{E D}$ \\
\hline Subtração & $(m, a, b)_{E D}-(n, c, d)_{D E}=(m-n, a+d, b+c)_{E D}$ \\
\hline Multiplicação por escalar & $(m, a, b)_{E D} x(n, 0,0)=(m n, a n, b n)$ \\
\hline \multicolumn{2}{|l|}{ Multiplicação por fuzzy } \\
\hline Para $m>0$ e $n>0$ & $(m, a, b)_{E D} \otimes(n, c, d)_{E D} \approx(m n, c m+a n, d m+b n)_{E D}$ \\
\hline Para $m<0$ e $n>0$ & $(m, a, b)_{D E} \otimes(n, c, d)_{E D} \approx(m n, a n-d m, b n-c m)_{D E}$ \\
\hline Para $\mathrm{m}<0$ e $\mathrm{n}<0$ & $(m, a, b)_{E D} \otimes(n, c, d)_{E D} \approx(m n,-b n-d m,-a n-c m)_{D E}$ \\
\hline Inverso para $(\mathrm{m}>0)$ & $(m, a, b)_{E D}^{-1} \approx\left(m^{-1}, b m^{-2}, a m^{-2}\right)_{D E}$ \\
\hline
\end{tabular}

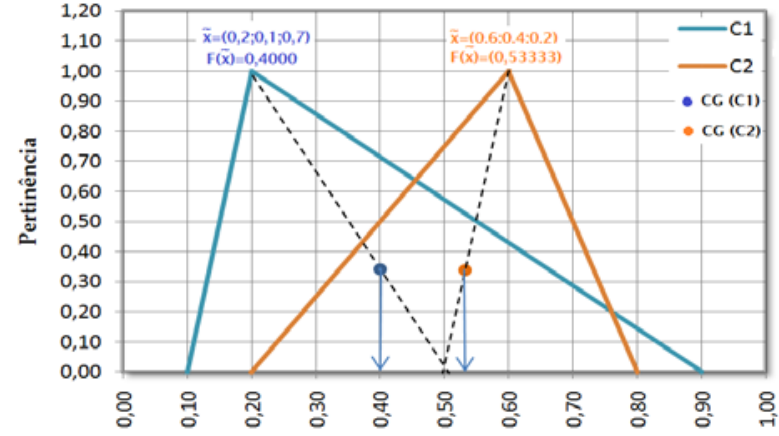

Figura 3 - Comparação dos números fuzzy $(0.6 ; 0.4 ; 0.2)$ e $(0.2 ; 0.1 ; 0.7)$.

\section{DESCRIÇ̃̃O DE CRITÉRIOS FUZZY}

Os problemas de planejamento ambiental e de planejamento de recursos hídricos envolvem critérios que possuem definições aproximadas, baseadas em estimativas.

Muitas dessas estimativas são descritas por valores médios, ou baseados em projeções, probabilidades ou tendências, que carregam diferentes graus de imprecisão. Nesses casos, os critérios são melhores representados por faixas prováveis de valores, com valores de saturação correspondente a estimativa tradicional, mas com intervalos possíveis de variação para a direita e/ou para esquerda desse valor principal.

A própria definição dos limites fiduciais em distribuições estatísticas descreve uma faixa de variação para o valor da estimativa. Esse valor poderá assumir qualquer valor real dentro desse intervalo com a mesma probabilidade em um intervalo (1- $) \%$ de confiabilidade. Nesse caso a representação do critério também é possível pela adoção de números fuzzy.

São vários os critérios que podem ser representados por números fuzzy, mesmo os mais tradicionalmente utilizados e considerados como estimativas precisas. Os critérios que poderiam ser representados por números fuzzy são de diferentes naturezas e podemos citar alguns exemplos a seguir:

1. Crescimento populacional- Diferentes projeções de crescimento populacional provenientes de uma mesma amostra, o que dificulta a escolha de um valor, existirá sempre uma incerteza de qual estimativa é a mais indicada;

2. Qualidade de água - estimada por meio de indicadores coletados com frequência média bimestral e dependem das vazões dos cursos d'água. As classes de enquadramento são definidas por faixas e as linhas que as separam são tênues e não podem ser consideradas como precisa, sendo, então, fuzzy;

3. Disponibilidade hídrica - baseada em dados hidrológicos como curva de permanência proveniente de tratamento estatístico, o que também carrega certa imprecisão;

4. Qualidade de vida - parâmetro bastante subjetivo, baseado em um conjunto de outros indicadores cuja interpretação demanda subjetividade do analista; assim, sua definição carece de precisão matemática;

5. Taxa de crescimento econômico - baseado em estimativas e indicadores econômicos. A realidade tem mostrado a fragilidade dessas estimativas, que são baseadas em premissas 
básicas como taxas de juros fixas, previsão de investimentos, taxas de inflação previstas, que não são exatas;

6. Geração de empregos ou renda - baseado em estatísticas de atividades potenciais e indicadores econômicos, que também carregam subjetividade nas estimativas;

7. Análise benefício/custo - o mais tradicional de todos os critérios, também é determinado por análises e projeções que podem não ser realista, baseado em taxas de retorno, valor presente líquido sobre as receitas, preços de insumos, custo de manutenção e operação, que podem variar muito. Muitas das hipóteses dos cálculos podem não se concretizar plenamente, comprometendo o desempenho do projeto em questão.

O que podemos concluir é que em boa parte dos critérios, que atualmente consideramos de natureza exclusivamente técnica, carrega alguma subjetividade, pois são assumidas hipóteses baseadas em estimativas que não são exatas, mas valores médios com intervalos de variação, mesmo que pequenos. Uma ferramenta que permita incorporar a fragilidade dessas estimativas para os critérios vem a ser bastante útil.

\section{AVALIAÇÃO DE ALTERNATIVAS}

A avaliação dos métodos propostos Fuzzy-CP e Fuzzy-CGT, esses foram aplicados por meio de um estudo de caso. Optou-se por utilizar um estudo já realizado com análise multicriterial, o qual não havia utilizado a abordagem fuzzy. Nesse caso, foi necessária a introdução de algumas modificações no estudo de caso original apresentado por Alves (2003).

No estudo desenvolvido por Alves (2003) foram selecionados possíveis sítios para instalação de ETE. Com os seis sítios com os maiores potenciais, procurou-se analisar, em uma segunda fase, o conjunto Sítio/Tecnologia que possibilitaria a seleção da alternativa de melhor compromisso. Analisou quatro tecnologias distintas de tratamento de esgotos, o que possibilitou criar alguns planos de ação com a mesma tecnologia de tratamento, porém em sítios distintos.

Os pesos atribuídos aos critérios também diferem daqueles utilizados por Alves (2003), que utilizou valores médios obtidos de um questionário estruturado (DELPHI), e que possuíam pouca varia- ção entre si. Na aplicação do método DELPHI não é raro na definição dos pesos, por meio de questionários aos especialistas, haver pouca diferença entres os critérios considerados como o de maior e o de menor pesos.

As importâncias relativas entre os critérios vão sendo diminuídas, na medida em que, são descartados os valores que estão fora do intervalo definido pelo $1^{-}$e $3^{\circ}$ Quartis, aproximando todos os valores atribuídos aos critérios para uma mesma faixa. Esse pequeno range que resulta da aplicação do método DELPHI deturpa o real grau relativo de importância entre os critérios, pois na maioria das aplicações os valores máximos e mínimos acabam ficando em uma faixa de valores estreita compreendida entre 7 e 9 (em uma escala de 1 a 10), com raras exceções.

A partir dos valores dos pesos atribuídos no estudo de Alves (2003), procedeu-se a uma redefinição dos pesos com a utilização da metodologia de determinação de pesos do método AHP (Analytic Hierarch Process), Saaty (1980). Trata-se de uma pequena adaptação.

Primeiramente, ordenam-se os critérios do maior para o menor, logo em seguida monta-se uma matriz n x n (critério x critério) e calculam-se as diferenças relativas entre o critério $i$ com os demais, de menor importância, depois entre o critério $i+1$ com os demais de menor importância, e assim sucessivamente.

O passo seguinte é substituir essas diferenças com os números que definem a escala de Saaty (1980) que varia de 1 a 9, montando-se uma matriz diagonal superior com essa escala. A partir daí define-se os pesos pelo procedimento do método AHP, preenchendo a matriz inferior com o preenchimento da coluna com o inverso dos valores da linha. $\mathrm{O}$ resultado é muito próximo àquele definido pela aplicação do método MACBETH (Bana e Costa e Vansnick, 1995 e 1997).

Os resultados obtidos por este trabalho não poderão ser comparados com aqueles obtidos por Alves (2003), uma vez que possuem cenários de pesos diferentes, como também não adotou intervalos fuzzy em sua matriz de avaliação, impossibilitando uma comparação direta dos resultados.

Os critérios analisados foram:

1. Distância: Analisada pela distância entre os pontos de descarga dos coletores de esgotos nos cursos d'água e os sítios, adotado critério fuzzy uma vez que os custos são aproximados e, geralmente subestimados; 
2. Altitude: Avaliado pela diferença entre a altitude dos pontos de descarga dos coletores de esgotos e a altitude média em cada sítio (visando evitar o bombeamento dos efluentes a maiores alturas, para limitar o gasto com equipamentos de bombeamento, de condutos e de energia elétrica), adotado critério escalar;

3. Declividade: Avaliado pela declividade média em cada sítio (visando evitar grandes movimentos de terra como cortes e aterros, que alimentam os processos de erosão do solo e assoreamento de cursos d'água, além do aumento dos custos de implantação). Foram adotados critérios fuzzy, uma vez que os sítios não são planos, e possuem declividades de até $15 \%$;

4. Área: Analisado pelo requisito de área para tipo de tratamento de esgoto adotado nas alternativas (grandes áreas representam custos elevados de desapropriação, áreas pequenas inviabilizam algumas tecnologias que demandam espaço físico), adotado critério escalar;

5. Potência: Avaliado a partir de potência total instalada requerida pela operação para as tecnologias de tratamento adotado nas alternativas. Adotado critério escalar;

6. Custo de Implantação: Avaliado pelo custo de implantação de cada tecnologia adotado nas alternativas. Foi assumido como critério fuzzy, pois as estimativas de custo geralmente não são precisas, por não ser possível prever todas as necessidades específicas a serem encontradas em cada sítio, tais como: profundidade e resistência do solo, instabilidades, ou qualquer outro problema de determinação sem prévios ensaios de campo e análises de laboratório;

7. Custo de Operação e Manutenção: Avaliação relativa entre as diversas tecnologias de tratamento, previsão de quantidade de insumos, preços, variação de preços, etc., que não são fixos e não têm a mesma variação anual. Adotado critério fuzzy;

8. Tempo Hidráulico de Detenção: Avaliado pelo tempo médio de detenção hidráulica de cada tecnologia de tratamento analisada, utiliza-se o tempo médio de percurso. Estimativa de tempo pode ser considerada como critério fuzzy;

9. Confiabilidade do Sistema: Avaliado de forma relativa entre as diversas tecnologias, Adotado critério fuzzy.
10. Simplicidade de Operação e Manutenção: Avaliado de forma relativa entre as diversas tecnologias. Toda avaliação relativa baseada em estimativas não é fixa. Adotado como critério fuzzy;

11. Quantidade de Lodo a ser Tratado: Analisado por meio das quantidades de lodo a ser tratado. O volume de lodo produzido é um valor médio, depende das características do efluente, que variam de acordo com a localização, clima e classe social na qual é produzido. Estimativa, adotado critério fuzzy;

12. Eficiência na Remoção (DBO, N, P e Coliformes): Avaliado de forma relativa entre as diferentes tecnologias de tratamento de esgoto. Estimativa, adotado critério fuzzy;

13. Qualidade de Água: Avaliado pela proximidade do sítio (lançamento do efluente tratado) em um ponto de qualidade inferior no curso d'água. Considerando o lançamento em ponto do rio a montante da ETE, em que a poluição da água é pior que a do efluente tratado. Adotado critério fuzzy.

14. Zoneamento Municipal: Avaliado pela restrição de implantação da ETE em determinadas zonas municipais. Adotado critério escalar, pois utilizou-se o mapa de zoneamento da cidade, que define as zonas possíveis ou não para esta atividade. Definida por Lei municipal;

15. Menor possibilidade de Problemas Ambientais: Avaliado de forma relativa entre as diferentes tecnologias, e considera expectativas de ocorrência e não é exata, é uma avaliação subjetiva. Adotado como critério fuzzy.

As alternativas foram:

- Alternativa 1: Combina o sítio melhor classificado com a tecnologia de reatores anaeróbios.Requer uma área de 0,05 a 0,10 $\mathrm{m}^{2} /$ hab e para atender a população de projeto seria necessária uma área de 0,38 a 0,76 ha;

- Alternativa 2: Combina o segundo melhor sítio com a utilização da tecnologia de lodos ativados com aeração prolongada. Requer uma área de 0,25 a $0,35 \mathrm{~m}^{2} /$ hab e para atender a população seria necessária uma área de 1,91 a 2,67 ha;

- Alternativa 3: Combina o terceiro melhor sítio com a tecnologia de lagoa aerada facultativa. Requer uma área de 0,25 a 0,5 
$\mathrm{m}^{2} /$ hab e, nesse caso, necessitaria de uma área compreendida entre 1,91 a 3,81 ha;

- Alternativa 4: Combina o quarto melhor sítio com a tecnologia de filtro biológico e baixa carga, que requer uma área de 0,2 a $0,6 \mathrm{~m}^{2} /$ hab. Para essa tecnologia a área necessária seria de 1,53 a 2,29 ha;

- $\quad$ Alternativa 5: Combina o quinto melhor sítio com a tecnologia de lagoa aerada de mistura completa. Requer uma área de $0,2 \mathrm{a}$ $0,5 \mathrm{~m}^{2} / \mathrm{hab}$, e para atender a população a área requerida deve estar compreendida entre 1,53 a 3,81 ha;

- Alternativa 6: Adota o sexto melhor sítio combinado com a tecnologia de reatores anaeróbios e requer uma área de 0,05 a 0,10 $\mathrm{m}^{2} /$ hab, o que equivale a uma área de 0,38 a 0,76 ha.

Os intervalos fuzzy adotados variaram de $0 \mathrm{a}$ $25 \%$ da nota atribuída para cada uma das alternativas, dependendo do critério. O Quadro 1 apresenta os valores para cada uma das alternativas, para cada um dos critérios considerados. As colunas representadas pelo símbolo "\#” correspondem aos valores principais dos critérios, enquanto as colunas correspondentes às letras " $\mathrm{E}$ " e "D", apresentam os intervalos, esquerdo e direito, do valor principal, que indicam sua possibilidade de variação no intervalo definido por (\# - E, \# + D) .

O escopo deste artigo é o de apresentar ferramentas capazes de trabalhar com critérios que carregam certa subjetividade. O estudo de caso original, adotado para exemplificar os métodos propostos, não foi originalmente definido como problema fuzzy. Assim sendo, para a validação dos métodos propostos, os intervalos dos valores fuzzy para alguns critérios foram definidos arbitrariamente.

Apresenta-se, como exemplo de cálculo dos intervalos fuzzy, o critério 12 (Eficiência de Remoção). Esse critério originalmente foi composto com 4 indicadores (DBO, N, P e Coliformes) no trabalho de Alves (2003). Para ETEs constituídas por fossas sépticas seguidas de filtro anaeróbio, apresentam na literatura uma eficiência de remoção da ordem de 80 a $85 \%$, e os verificados nas ETEs estudadas uma variação entre 36 a $82 \%$ (Oliveira e Von Sperling, 2005, pág. 354). Desta forma, pode-se adotar o valor médio da literatura como sendo o valor principal, e os intervalos fuzzy, como sendo o menor e a maior eficiência. Neste caso o valor da eficiência variou entre 36 a $85 \%$, sendo o valor médio igual a $82,5 \%$, não sendo um valor simétrico, tendendo muito mais a eficiência menores do que maiores. A partir dessa informação, utilizam-se as funções de valores para traduzir os valores das eficiências em valores dos critérios. Esse valor corresponde somente ao parâmetro DBO, os demais devem ser definidos para compor o valor final do critério, para cada uma das tecnologias.

Para o critério de número 6 (custo de implantação) utilizou-se um intervalo de 5 a $20 \%$ para maior, e $0 \%$ para menor, uma vez que em projetos de engenharia em geral não se observa o custo de uma obra sair mais barato que o previsto, mas sempre mais caro. Desta forma, as ETEs com maiores tecnologias serão esperados maiores erros de estimativas de custo, o que fará com que os intervalos fuzzy a esquerda serão maiores, como punição ao aumento deste custo.

Para verificar a coerência das respostas realizou-se uma análise de sensibilidade aos dados do problema, acrescentando-se ou subtraindo-se $10 \%$ do valor do peso do critério considerado como o mais importante, critério de número 12 (Eficiência de Remoção).

\section{RESULTADOS PARA OS DADOS FUZZY}

Os valores obtidos pela aplicação dos dois métodos fuzzy estão apresentados na Tabela 2. Nessa tabela, as performances das seis alternativas estão expressas de duas maneiras simultâneas: as colunas (b) e (g) apresentam o valor da distância, que correspondem aos resultados das análises multicriteriais, sem a consideração dos intervalos fuzzy, o que torna o problema escalar.

As colunas (c) e (h) apresentam os intervalos a esquerda, dos resultados escalares, da implementação do método multicriterial, para os números principais, para cada uma das alternativas. As colunas (d) e (i) apresentam os intervalos direitos desses números principais.

Os resultados da análise multicriterial, considerando-se os intervalos fuzzy, são apresentados nas colunas (e) e (j), que representam as distâncias "defuzzificadas". Essas foram obtidas pelas alternativas considerando-se os critérios representados por intervalos fuzzy.

A “defuzzificação" proposta por Yager (1981) retorna o mesmo valor do número principal caso os intervalos fuzzy sejam simétricos. Há na literatura diferentes propostas para a "escalarização" de números fuzzy, que podem fornecer resultados diferentes, mas possibilitam uma comparação escalar desses 
números, não necessariamente com os mesmos valores do principal fuzzy.

Os mesmos parâmetros para os critérios generalizados e os mesmos pesos foram utilizados na aplicação dos métodos fuzzy (CP e CGT). Os resultados líquidos e as classificações obtidas pela implementação dos intervalos fuzzy, também estão apresentadas na Tabela 2, pelas colunas (a) e (f).

Como mostrado na Tabela 2, as alternativas de melhores compromissos, definidas pelos métodos CGT e CP, em ambos os casos são, nesta ordem:

- A alternativa de número 1, que possui avaliação máxima em 7 critérios, e;

- A alternativa de número 3, com duas avaliações máximas (critérios representados pela matriz de avaliação definida pelo Quadro $1)$;

As alternativas de números 6 e 4 possuem seis e quatro critérios com notas máximas, respectivamente, mas no desempenho geral, perdem para a alternativa de número 3 , que apresentou melhor performance. Para a alternativa de número 1, os critérios décimo primeiro e décimo quinto foram os que definiram sua posição, devido aos pesos desses critérios serem maiores em relação aos demais.

Observa-se que a alternativa de número 2 foi classificada como a pior alternativa, mesmo possuindo o mesmo número de critérios com notas máximas que a alternativa de número 3, porém, os dois critérios que obtiveram notas máximas, possuem pesos bem inferiores àqueles com notas máximas da alternativa 3, classificada em segunda posição.

As Figuras 4 e 5 ilustram as distâncias fuzzy obtidas pelas alternativas pelos dois métodos multicritérios fuzzy aplicados, o Fuzzy-CP e o Fuzzy-CGT.

Pode-se observar que a introdução de critérios fuzzy não altera os valores das distâncias originais fornecidas pela aplicação do método multicriterial, porém, após a "defuzzificação", na ocorrência de grandes assimetrias, poderá haver alterações nas hierarquizações das alternativas. Existem algumas propostas de comparações diretas entre números fuzzy, mas ainda nenhuma consagrada. Em sua maioria, essas propostas são aplicadas para alguns casos especiais.

A adoção de critérios fuzzy, neste trabalho, não alterou a hierarquização das alternativas, mas alterou os valores das distâncias, calculadas pelos métodos, interferindo nas diferenças relativas entre essas distâncias, o que pode possibilitar a alteração das hierarquizações em outros estudos.

Procurou-se então avaliar se as classificações seriam alteradas com um teste de sensibilidade. Nesse teste foram rodados mais dois cenários de pesos atribuindo $\pm 10 \%$ ao critério de maior peso. Não foram observadas quaisquer alterações nas hierarquizações, para nenhum dos dois métodos, apenas alterações dos valores das distâncias dos métodos.

A escolha dos parâmetros expressa a preferência ou a opinião do decisor (DM), como previsto, influenciaram fortemente o resultado.

Considerando que as opiniões de diferentes pessoas raramente coincidem, de uma maneira geral pode-se afirmar que não existirá uma única solução aceita por todos, com isso, os resultados obtidos por este trabalho devem ser vistos sob esta perspectiva.

$\mathrm{O}$ método CGT mostrou-se mais sensível às variações devido à incorporação dos intervalos fuzzy que o método CP.

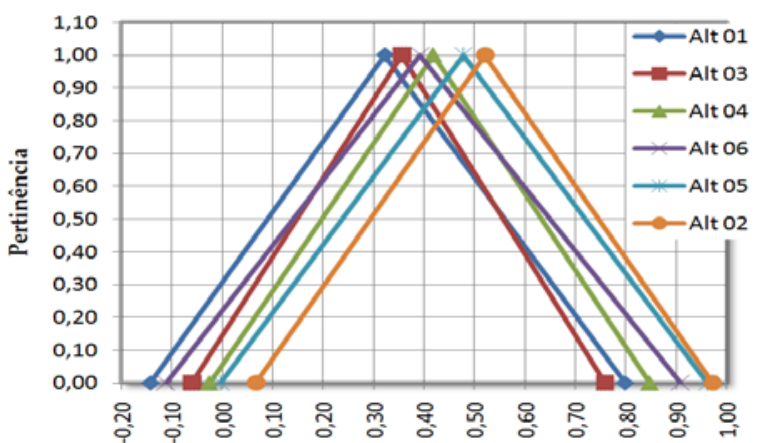

Figura 4 - Comparação dos resultados a aplicação do método $F u z z y-C P$ às 6 alternativas.

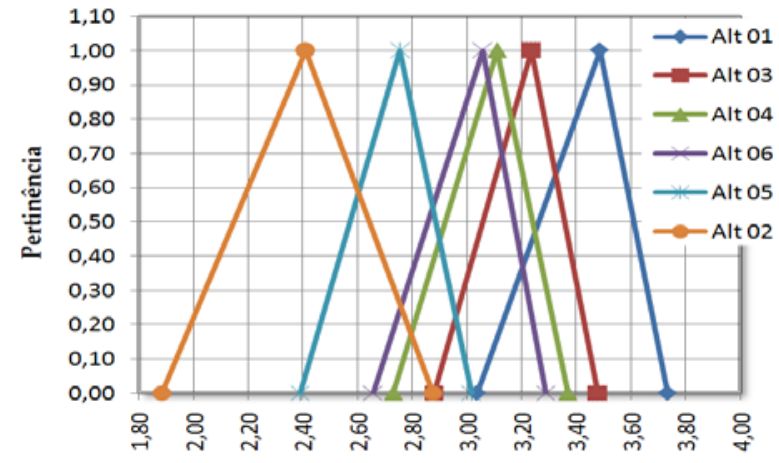

Figura 5 - Comparação dos resultados a aplicação do método Fuzzy-CGT às 6 alternativas. 
Incorporação de Matemática Fu₹zy em Métodos Multicriteriais para Descrever Critérios Subjetivos em Planejamento de Recursos Hídricos: Fuz:y - CP e Fuz:y - CGT

Quadro 1 - Matriz de avaliação de cinco alternativas hipotéticas de um problema de planejamento.

\begin{tabular}{|c|c|c|c|c|c|c|c|c|c|c|c|c|c|c|c|c|c|c|c|}
\hline \multirow[t]{2}{*}{ Crit } & \multirow[t]{2}{*}{ Pesos } & \multicolumn{3}{|c|}{ Alternativa 01} & \multicolumn{3}{|c|}{ Alternativa 02} & \multicolumn{3}{|c|}{ Alternativa 03} & \multicolumn{3}{|c|}{ Alternativa 04} & \multicolumn{3}{|c|}{ Alternativa 05} & \multicolumn{3}{|c|}{ Alternativa 06} \\
\hline & & $\#$ & $\mathrm{E}$ & $\mathrm{D}$ & $\#$ & $\mathrm{E}$ & $\mathrm{D}$ & $\#$ & $\mathrm{E}$ & $\mathrm{D}$ & $\#$ & $\mathrm{E}$ & $\mathrm{D}$ & $\#$ & $\mathrm{E}$ & $\mathrm{D}$ & $\#$ & $\mathrm{E}$ & $\mathrm{D}$ \\
\hline 1 & 3.01 & 10.0 & 1.0 & 0.2 & 9.8 & 1.0 & 0.2 & 9.1 & 0.9 & 0.5 & 8.8 & 0.9 & 0.5 & 8.7 & 0.9 & 0.5 & 7.7 & 0.8 & 0.5 \\
\hline 2 & 2.77 & 7.3 & 0.0 & 0.0 & 6.1 & 0.0 & 0.0 & 4.7 & 0.0 & 0.0 & 4.7 & 0.0 & 0.0 & 4.2 & 0.0 & 0.0 & 8.1 & 0.0 & 0.0 \\
\hline 3 & 2.70 & 10.0 & 1.0 & 0.2 & 10.0 & 1.0 & 0.4 & 9.8 & 1.0 & 0.3 & 10.0 & 1.0 & 0.5 & 10.0 & 1.0 & 0.5 & 7.9 & 0.8 & 0.5 \\
\hline 4 & 3.22 & 10.0 & 0.0 & 0.0 & 6.0 & 0.0 & 0.0 & 4.0 & 0.0 & 0.0 & 7.0 & 0.0 & 0.0 & 5.0 & 0.0 & 0.0 & 10.0 & 0.0 & 0.0 \\
\hline 5 & 3.46 & 10.0 & 0.0 & 0.0 & 5.0 & 0.0 & 0.0 & 8.0 & 0.0 & 0.0 & 10.0 & 0.0 & 0.0 & 8.0 & 0.0 & 0.0 & 10.0 & 0.0 & 0.0 \\
\hline 6 & 4.53 & 8.0 & 1.6 & 0.0 & 4.0 & 0.4 & 0.0 & 9.0 & 0.45 & 0.0 & 3.0 & 0.3 & 0.0 & 9.0 & 1.35 & 0.0 & 8.0 & 1.6 & 0.0 \\
\hline 7 & 6.51 & 5.0 & 0.5 & 0.5 & 1.0 & 0.5 & 0.9 & 4.0 & 0.4 & 0.6 & 3.0 & 0.5 & 0.7 & 3.0 & 0.5 & 0.7 & 5.0 & 0.5 & 0.5 \\
\hline 8 & 2.87 & 10.0 & 1.0 & 0.4 & 10.0 & 1.0 & 0.5 & 3.0 & 0.5 & 0.7 & 10.0 & 1.0 & 0.5 & 5.0 & 0.5 & 0.5 & 10.0 & 1.0 & 0.5 \\
\hline 9 & 8.55 & 3.0 & 0.5 & 0.7 & 5.0 & 0.5 & 0.5 & 4.0 & 0.4 & 0.6 & 4.0 & 0.4 & 0.6 & 3.0 & 0.5 & 0.7 & 3.0 & 0.5 & 0.7 \\
\hline 10 & 3.82 & 4.0 & 0.4 & 0.6 & 2.0 & 0.5 & 0.8 & 4.0 & 0.4 & 0.6 & 3.0 & 0.5 & 0.7 & 3.0 & 0.5 & 0.7 & 4.0 & 0.4 & 0.6 \\
\hline 11 & 7.44 & 10.0 & 0.5 & 0.3 & 1.0 & 0.7 & 0.5 & 10.0 & 0.7 & 0.5 & 5.0 & 0.5 & 0.4 & 10.0 & 0.7 & 0.5 & 10.0 & 0.7 & 0.5 \\
\hline 12 & 10.00 & 6.0 & 1.5 & 0.3 & 8.0 & 2.0 & 0.4 & 10.0 & 2.5 & 0.5 & 10.0 & 2.5 & 0.5 & 10.0 & 2.5 & 0.5 & 6.0 & 1.5 & 0.3 \\
\hline 13 & 5.09 & 8.0 & 0.8 & 0.5 & 8.0 & 0.8 & 0.5 & 6.0 & 0.6 & 0.4 & 6.0 & 0.6 & 0.4 & 6.0 & 0.6 & 0.4 & 10.0 & 1.0 & 0.5 \\
\hline 14 & 3.79 & 5.0 & 0.0 & 0.0 & 7.0 & 0.0 & 0.0 & 7.0 & 0.0 & 0.0 & 7.0 & 0.0 & 0.0 & 7.0 & 0.0 & 0.0 & 1.0 & 0.0 & 0.0 \\
\hline 15 & 5.74 & 10.0 & 1.5 & 0.5 & 8.0 & 1.2 & 0.4 & 6.0 & 0.9 & 0.3 & 8.0 & 1.2 & 0.4 & 4.0 & 0.6 & 0.2 & 10.0 & 1.5 & 0.5 \\
\hline
\end{tabular}

Tabela 2 - Hierarquização das alternativas expressas como números fuzzy.

\begin{tabular}{|c|c|c|c|c|c|c|c|c|c|c|}
\hline \multirow{3}{*}{$\begin{array}{l}\text { Classifi- } \\
\text { cação }\end{array}$} & \multicolumn{5}{|c|}{ Cooperative Game Theory (CGT) } & \multicolumn{5}{|c|}{ Compromise Programming (CP) } \\
\hline & (a) & (b) & (c) & $(d)$ & (e) & $(f)$ & (g) & (h) & (i) & (j) \\
\hline & Alter. & (CGT , & $\mathrm{E}$ & D) & Defuzzificação & Alter & $(\mathrm{CP}$ & $\mathrm{E}$ & D) & Defuzzifcação \\
\hline $1^{\mathrm{a}}$ & 1 & $(3.486$ & 0.449 & $0.248)$ & 3.419 & 1 & $(0.322$ & 0.465 & $0.476)$ & 0.326 \\
\hline $2^{\mathrm{a}}$. & 3 & $(3.235$ & 0.354 & $0.243)$ & 3.198 & 3 & $(0.356$ & 0.416 & $0.403)$ & 0.352 \\
\hline $3^{\mathrm{a}}$. & 4 & $(3.112$ & 0.381 & $0.259)$ & 3.072 & 6 & $(0.392$, & 0.504 & $0.516)$ & 0.396 \\
\hline $4^{\mathrm{a}}$. & 6 & (3.057, & 0.401 & $0.233)$ & 3.001 & 4 & (0.418, & 0.443 & $0.429)$ & 0.413 \\
\hline $5^{\mathrm{a}}$ & 5 & (2.757, & 0.366 & $0.260)$ & 2.722 & 5 & (0.478, & 0.481 & $0.484)$ & 0.479 \\
\hline $6^{\mathrm{a}}$. & 2 & (2.410, & 0.525 & $0.446)$ & 2.383 & 2 & (0.521, & 0.454 & $0.451)$ & 0.520 \\
\hline
\end{tabular}

Esse comportamento pode ser esperado, uma vez que no método CGT a matemática envolvida na agregação é a multiplicativa, enquanto que no método CP os intervalos fuzzy a agregação é a aditiva. As diferenças entre os resultados do método CGT, com ou sem dados fuzzy, são maiores se comparados com aqueles originados pelo método CP.

A metodologia mostrou-se adequada para aplicações que envolvam critérios ambientais e sociais e que carregam certa subjetividade e a sua representação por meio de números fuzzy mostrou-se viável.

Critérios que não sejam ambientais ou sociais, mas que podem representar probabilidade ou algum tipo de subjetividade ou carregar alguma abstração também poderão ser descritos por números fuzzy. Esses critérios são, então, representados por meio de intervalos de possibilidades, definidos por faixas que podem ser representadas pelos números fuzzy.

Esta possibilidade introduz novas informações ao processo de tomada de decisão, resultando em uma hierarquização mais científica para a consideração da subjetividade, em que a imprecisão dos dados é então considerada e avaliada.

Assim, a incorporação de aritmética fuzzy mostrou-se viável nos métodos CP e CGT, e a incorporação da matemática fuzzy em outros métodos multicriteriais também pode vir a ser viável. Contribuindo na construção de ferramentas que enriqueçam o processo de tomada de decisão multicriterial e, qualquer tipo de planejamento, mas principalmente nos de planejamento ambiental e de recursos hídricos, por envolverem critérios ambientais e sociais. 
Observa-se, porém, que a adoção dos intervalos fuzzy deve ser realizada com o maior rigor científico possível. Isto porque, com a análise multicriterial fuzzy, as incertezas definidas pelos intervalos (esquerdo e direito) dos números fuzzy serão somados, multiplicados, mas nunca subtraídos ou divididos. E no final do processo de cálculo esses intervalos terão crescido, pois representarão o acúmulo de todas as incertezas consideradas na valoração dos critérios.

Demonstra-se, desta forma, que é necessário refinar o processo de valoração dos critérios, diminuindo-se ao máximo a faixa dessas incertezas, para não comprometer os resultados finais, mas sim, melhorar não só os resultados dos métodos, mas também o processo de decisão.

Recomenda-se, para futuros estudos a adoção de outros métodos de comparação dos números fuzzy, sejam eles por meio da "defuzzificação", ou por comparação direta entre esses números.

\section{AGRADECIMENTOS}

$\mathrm{O}$ autor agradece a Fundação de Amparo à Pesquisa de São Paulo (FAPESP) pela bolsa de pósdoutorado, realizada na Universidade de Toronto, Canadá, PROCESSO FAPESP \#1999/10268-1, e bolsa de mestrado do aluno Fabrício Vieira Alves, PROCESSO FAPESP \# 2000/09624-7.

\section{REFERÊNCIAS}

ALVES, F. V. (2003) - Seleção de sítio e tecnologia para estação de tratamento de esgoto por meio de SIG e métodos multicriteriais. Estudo de caso: Paulínia SP. Dissertação de mestrado, FEC-UNICAMP, 200 p.

BANA E COSTA, C.A., VANSNICK, J.C.,1995, Uma nova abordagem ao problema da construção de uma função de valor cardinal: MACBETH, Investigação Operacional, 15 pp. 15-35.

BANA E COSTA, C.A., VANSNICK, J.C.,1997, Thoughts on a theoretical framework for measuring attractiveness by categorical based evaluation technique (MACBETH). In: Clímaco, J. (ed.), Multicriteria Analysis, Springer-Verlag, Berlin.

BOCLIN, A. de S. C. e MELLO, R. de (2006) - A decison support method for environmental impact assess- ment using a fuzzy logic approach. Ecological economics, 58, pags. 170-181.

DUBOIS, D. e PRADE, H. (1978) - Operational on fuzzy numbers. International Journal of Systems Science (24), 228-238.

DUBOIS, D. e PRADE, H. (1979) - Fuzzy real algebra: Some results. Fuzzy Sets and Systems. 2, 327-348.

DUBOIS, D. e PRADE, H. (1984) - Criteria aggregation and ranking of alternatives in the framework of fuzzy set theory. Studies in the Management sciences, 20, pp. 209-240.

DUBOIS, D. e PRADE, H. (1988) - Possibility theory. Springer. New York. 280 p.

ERGAS, H. e ROBSON, A. (2009) - The social losses from inefficient infrastructure projects: recent australian experience. Productivity commission round table. Strengthening evidence-based policy in the Australian federation, 17-18 august 2009.

EUSTÁCHIO, J. A. V. e TÁVORA JÚNIOR, J. L. (1999) Metodologias de avaliação de ativos ambientais: Uma comparação entre medidas. In: III Encontro Nacioanl da ECOECO. Sociedade Brasileira de Economia Ecológica. Recife-PE.

GERSHON, M. e DUCKSTEIN, L. (1983) - Multiobjectives approaches to river basin planning. Journal of water planning and management, 109(1), pages 13-28.

GOUMAS, M. e LYGEROU, V. (2000) - "An extension of the PROMETHEE method for decision making in fuzzy environment: Ranking of alternative energy exploitation projects". European Journal of Operational Research, (123), 606-613.

HOYOS, D. (2010) - The state of the art of environmental valuation with discrete choice experiments. Ecological economics, 69, 1595-1603.

JARDIM, S. B. e LANNA, A. E. L. (2003) - Aplicabilidade de algumas técnicas de análise multiobjetivo ao processo decisório no âmbito dos comitês de gerenciamento de bacia hidrográfica. Revista Brasileira de Recursos Hídricos, vol 8 (4), 169-191.

MUNDA, G. (2008) - Social Multi-Criteria Evaluation for a Sustainable Economy. Springer-Verlag Berlin Heidelberg, $210 p$.

MUNIER, N. (2006) - Economic growth and sustainable development: could multicriteria analysis be used to solve this dichotomy? Environment, Development and Sustainability, 8: 425-443.

OLIVEIRA, S. M. A. C. e VON SPERLING, M. (2005) - Avaliação de 166 ETEs em operação no País, compreendendo diversas tecnologias. Parte I - Análise de desempenho. Engenharia Sanitária e Ambiental, vol.10, no. 4, pags. 347-357.

SAATY, T. L. The analytic hierarchy process: planning, priority setting, resource allocation. New York, London: McGraw-Hill, 1980. 
Incorporação de Matemática Fu₹zy em Métodos Multicriteriais para Descrever Critérios Subjetivos em Planejamento de Recursos Hídricos: Fuzzy - CP e Fuzzy - CGT

SHARRATT, P. (1999) - Environmental criteria in design. Computers \& chemical engineering. 23, 1469-1475.

YAGER, L. A. (1965) - A procedure for ordering fuzzy subsets of the unit interval. Information Science (24), 143161.

ZELENY, M. (1982) - Multiple criteria decison making. McGraw-Hill Book Company. New York, 563p.

ZUFFO, A. C. (2001) - Incorporação de Lógica Nebulosa ao Planejamento Ambiental de Recursos Hídricos: Uma Abordagem Multicriterial. Relatório de pesquisa do programa de aperfeiçoamento no exterior realizado na "University of Toronto" (UofT), Ontário, Canadá. Processo FAPESP \#1999/10268-1.

ZUFFO, A. C. (2009) - Fuzzy set to represent environmental and social criteria in a multicriteria approach to water resources planning. In: ANAIS ASCE, 33rd International IAHR Congress: Water Engineering for a SuStainable Environment. Vancouver, Canada, August.

Use of Fuzzy Math in Multicriteria Methods to Describe Subjective Criteria in Water Resources Planning

\section{ABSTRACT}

In many environmental and water resources planning and management studies it is necessary to use criteria with few data or whose interpretation relies on the knowledge of specialists. For this reason, criteria that are based on subjectivity and scalar numbers do not fully represent them. Social and environmental criteria are assessed based on estimations or uncertainties; they can be better represented by a range of values. Fuzzy numbers better describe some social and environmental criteria, since they can easily incorporate subjectivity. In general, fuzzy sets allow the expression of values or set of values for the criteria in percentage or in a small range of possibilities and, also, allow incorporating the experts' experience. This paper addresses the modification of two different multi-criteria methods know as "Cooperative Game Theory" (CGT) and "Compromise Programming" (CP), in order to work with fuzzy numbers. These methods were applied to selected sites and waste water treatment technology in the city of Paulinia - SP, Brazil. Fifteen criteria were considered to analyze six alternatives. The proposed fuzzy incorporation of social, environmental and economical criteria proved feasible and reliable, improving the decision making process.

Key-words: Water resources planning, fuzzy multicriteria methods, multicriteria analysis 